DESY 05-146, MZ-TH/05-16, hep-ph/0508129

\title{
Reconciling open charm production at the Fermilab Tevatron with QCD
}

\author{
B.A. Kniehl, ${ }^{1}$ G. Kramer, ${ }^{1}$ I. Schienbein, ${ }^{1}$ and H. Spiesberger ${ }^{2}$ \\ 1 II. Inst. für Theor. Physik, Univ. Hamburg, \\ Luruper Chaussee 149, 22761 Hamburg, Germany \\ 2 Institut für Physik, Johannes-Gutenberg-Universität, \\ Staudinger Weg 7, 55099 Mainz, Germany
}

(Dated: February 2, 2008)

\begin{abstract}
We study the inclusive hadrodroduction of $D^{0}, D^{+}, D^{*+}$, and $D_{s}^{+}$mesons at next-to-leading order in the parton model of quantum chromodynamics endowed with universal non-perturbative fragmentation functions (FFs) fitted to $e^{+} e^{-}$annihilation data from CERN LEP1. Working in the general-mass variable-flavor-number scheme, we resum the large logarithms through the evolution of the FFs and, at the same time, retain the full dependence on the charm-quark mass without additional theoretical assumptions. In this way, the cross section distributions in transverse momentum recently measured by the CDF Collaboration in run II at the Fermilab Tevatron are described within errors.
\end{abstract}


Recently, there has been much interest in the study of charmed-hadron $\left(X_{c}\right)$ production at hadron colliders, both experimentally and theoretically. The CDF Collaboration measured the differential cross sections $d \sigma / d p_{T}$ for the inclusive production of $D^{0}, D^{+}, D^{*+}$, and $D_{s}^{+}$ mesons (and their antiparticles) in $p \bar{p}$ collisions at the Fermilab Tevatron (run II) as functions of transverse momentum $\left(p_{T}\right)$ in the central rapidity $(y)$ region [1]. Unfortunately, the most advanced theoretical predictions available so far [2, 5], based on quantum chromodynamics (QCD) at next-to-leading order (NLO), consistently undershoot all the $D^{0}, D^{+}$, and $D^{*+}$ data by significant amounts, as is evident from Fig. 3 of Ref. [1], while no predictions for $D_{s}^{+}$mesons exist yet. It is presently an open question if this discrepancy is related to an experimental problem, a technically deficient QCD prediction, or the appearance of new physics beyond the standard model. Such a situation is familiar from inclusive bottomflavored-hadron $\left(X_{b}\right)$ production in run I, where a long-standing discrepancy between CDF data [6] and certain NLO predictions of QCD were, in fact, interpreted as an indication for low-energy supersymmetry [7]. It is, therefore, an urgent task to deepen our understanding of the inclusive hadroproduction of charmed hadrons on the basis of QCD in order to render the theoretical predictions as reliable as possible, so as to establish a sturdy anchor for new-physics searches. This is even more important in view of future physics at the CERN Large Hadron Collider, where the continuum production of charmed hadrons will provide important backgrounds for numerous new-physics signals. This is the main motivation of this letter. Moreover, we provide the first NLO prediction for the CDF $D_{s}^{+}$data 1]. Prior to explaining our improved theoretical framework and describing our updated input, for the reader's quick orientation, we present a brief survey of the various NLO approaches adopted so far in the literature.

In the so-called massless scheme, also known as zero-mass variable-flavor-number (ZMVFN) scheme [4, 8], the conventional parton model approach implemented in the modified minimal subtraction $(\overline{\mathrm{MS}})$ scheme is adopted, assuming that the charm $(c)$ quark can be treated as massless, although its mass $m$ is certainly larger than the asymptotic scale parameter $\Lambda_{\mathrm{QCD}}$. In this approach, the $c$ quark occurs as an incoming parton, leading to contributions in addition to those where it is produced by an incoming gluon $g$ or a light $u, d$, or $s$ quark. The $c$ quark fragments into the charmed hadron similarly as the gluon and the light quarks with a fragmentation function (FF), which must be known from other processes. The well-known factorization theorem provides a straightforward procedure for 
systematic higher-order perturbative calculations. Due to the assumption that $m=0$, the predictions are reliable only for large values of $p_{T}$, with $p_{T} \gg m$, where powers of $m^{2} / p_{T}^{2}$ can be neglected. This approach has the advantage, however, that the potentially large logarithms of the type $\ln \left(p_{T}^{2} / m^{2}\right)$ are absorbed into the $c$-quark parton distribution functions (PDFs) of the colliding hadrons and into the FF for the transition $c \rightarrow X_{c}$. These logarithms are resummed through the Dokshitzer-Gribov-Lipatov-Altarelli-Parisi (DGLAP) evolution equations. Still, $m$ appears in the initial conditions of the $c$-quark PDF and FF. In this respect, the $c$-quark PDF and FF differ from the PDFs of the gluon and the light quarks.

In the so-called massive scheme, also called fixed-flavor-number (FFN) scheme 9], the number of active quark flavors in the initial state is fixed to $n_{f}=3$, and the $c$ quark only appears in the final state. The physical value of $m$ is explicitly taken into account together with the variable $p_{T}$, as if the two were of the same order. In this scheme, $m$ acts as a cutoff for the initial- and final-state collinear singularities. However, in NLO, terms proportional to $\ln \left(p_{T}^{2} / m^{2}\right)$ arise at large $p_{T}$ values from collinear gluon emission off $c$ quarks or from almost collinear branchings of gluons into $c \bar{c}$ pairs. For $p_{T} \gg m$, these terms spoil the convergence of the perturbative series. The FFN approach with $n_{f}=3$ is thus limited to a narrow $p_{T}$ range, reaching up to a few times $m$. The advantage of this scheme is that the $m^{2} / p_{T}^{2}$ power terms are fully taken into account.

Obviously, the ZM-VFN and FFN schemes are valid in complementary $p_{T}$ regions, and is desirable to combine them in a unified approach that enjoys the virtues of both schemes and, at the same time, is bare of their flaws, i.e. one that resums the large logarithms, retains the full finite- $m$ effects, and preserves the universality of the FFs. This is vital for a reliable and meaningful interpretation of the CDF data 1], which mostly populate the transition region. A first attempt to implement such an interpolating scheme is the so-called fixedorder next-to-leading-logarithm (FONNL) scheme, in which the conventional cross section in the FFN approach is linearly combined with a suitably modified cross section in the ZMVFN approach with perturbative FFs, using a suitable $p_{T}$-dependent weight function [5, 10]. In both finite-mass approaches, FFN and FONNL, the FFN cross sections are convoluted with a non-perturbative $c$-quark FF, adjusted to $e^{+} e^{-}$data, that is not subject to DGLAP evolution.

Here, we wish to advocate an approach that is much closer in spirit to the ZM-VFN scheme, but keeps all $m^{2} / p_{T}^{2}$ power terms in the hard-scattering cross sections, namely the 
general-mass variable-flavor-number (GM-VFN) scheme, which has recently been elaborated for the photo- 11] and hadroproduction [12, 13] of heavy-flavored hadrons. In this approach, one starts from the $p_{T} \gg m$ region and absorbs the large logarithms $\ln \left(p_{T}^{2} / m^{2}\right)$ into the $c$ quark PDF of the incoming hadrons and the FF for the $c \rightarrow X_{c}$ transition. After factorizing the $\ln m^{2}$ terms, the cross section is infrared safe in the limit $m \rightarrow 0$, and $n_{f}=4$ is taken in the strong-coupling constant $\alpha_{s}$ and the DGLAP evolution equations. The remaining $m$ dependence, i.e. the $m^{2} / p_{T}^{2}$ power terms, is retained in the hard-scattering cross sections. These terms are important in the intermediate $p_{T}$ region, where $p_{T} \gtrsim m$, and are expected to improve the precision of the theoretical predictions. The large logarithms are absorbed into the PDFs and FFs by subtraction of the collinearly (mass) singular terms. However, in order to define a unique factorization prescription, one also has to specify non-singular terms. This is done by requiring that, in the limit $p_{T} \rightarrow \infty$, the known ZM-VFN hardscattering cross sections are recovered. To achieve this, subtraction terms are derived by comparing the FFN theory in the limit $m \rightarrow 0$ with the ZM-VFN theory, implemented in the $\overline{\mathrm{MS}}$ factorization scheme. This matching procedure is useful, since all commonly used $c$-quark PDFs and FFs are defined in the ZM-VFN scheme. The latter can then be used consistently together with hard-scattering cross sections calculated in the GM-VFN scheme. The derivation of the subtraction terms is described in Ref. 13].

We note that our implementation of the GM-VFN scheme is similar to the AivazisCollins-Olness-Tung (ACOT) 14] scheme formulated for the initial state of fully inclusive deep-inelastic scattering. The extension of this scheme to the inclusive production of heavy partons was considered in Ref. [15], where the resummation of the final-state collinear logarithms was only performed to leading order (LO) and parton-to-hadron FFs were not included. A comprehensive discussion of the differences between our approach and Ref. 15] concerning the collinear subtraction terms may be found in Ref. [13].

We now describe our calculation of the differential cross section $d^{2} \sigma /\left(d p_{T} d y\right)$ of $p+\bar{p} \rightarrow$ $X_{c}+X$, where $X_{c}=D^{0}, D^{+}, D^{*+}, D_{s}^{+}$and $X$ comprises the residual final-state hadrons, at NLO in the GM-VFN scheme. A crucial ingredient entering this calculation are the nonperturbative FFs for the transitions $a \rightarrow X_{c}$, where $a=g, u, \bar{u}, d, \bar{d}, s, \bar{s}, c, \bar{c}$. For $X_{c}=D^{*+}$, such FFs were extracted at LO and NLO in the $\overline{\mathrm{MS}}$ factorization scheme with $n_{f}=5$ massless quark flavors several years ago [4] from the scaled-energy $(x)$ distributions $d \sigma / d x$ of the cross section of $e^{+}+e^{-} \rightarrow D^{*+}+X$ measured by the ALEPH [16] and OPAL [1]] 
Collaborations at CERN LEP1. Two of us [18] recently extended the analysis of Ref. [4] to include $X_{c}=D^{0}, D^{+}, D_{s}^{+}, \Lambda_{c}^{+}$by exploiting appropriate OPAL data [19]. Besides the total $X_{c}$ yield, which receives contributions from $Z \rightarrow c \bar{c}$ and $Z \rightarrow b \bar{b}$ decays as well as from lightquark and gluon fragmentation, the ALEPH and OPAL Collaborations separately specified the contribution due to tagged $Z \rightarrow b \bar{b}$ events yielding $X_{b}$ hadrons, which then weakly decay to $X_{c}$ hadrons. The contribution due to the fragmentation of primary $c$ quarks into $X_{c}$ hadrons approximately corresponds to the difference of these two measured distributions. To test the scaling violations of these FFs and also the separation of the $c \rightarrow X_{c}$ component, these FFs were employed to interpret the $x$ distributions of $e^{+}+e^{-} \rightarrow X_{c}+X$ for center-ofmass energy $\sqrt{S}=10.55 \mathrm{GeV}$ measured by the CLEO Collaboration 20] at LEPP CESR, with very encouraging results. Further details may be found in Ref. [18].

In Refs. [4, 18], the starting scales $\mu_{0}$ for the DGLAP evolution of the $a \rightarrow X_{c}$ FFs in the factorization scale $\mu_{F}^{\prime}$ are taken to be $\mu_{0}=2 m$, with $m=1.5 \mathrm{GeV}$, for $a=g, u, \bar{u}, d, \bar{d}, s, \bar{s}, c, \bar{c}$ and $\mu_{0}=2 m_{b}$, with $m_{b}=5 \mathrm{GeV}$, for $a=b, \bar{b}$. The FFs for $a=g, u, \bar{u}, d, \bar{d}, s, \bar{s}$ are assumed to be zero at $\mu_{F}^{\prime}=\mu_{0}$ and are generated through the DGLAP evolution to larger values of $\mu_{F}^{\prime}$. Since the effect of the gluon FF is important at Tevatron energies, as was found for $D^{*+}$ production in Ref. 12], we repeated the fits of the $X_{c}$ FFs for the choice $\mu_{0}=m, m_{b}$. This changes the $c$-quark FFs only marginally, but has a strong effect on the gluon FF. For shortage of space, these new FFs will be presented elsewhere.

The calculation of the cross section $d^{2} \sigma /\left(d p_{T} d y\right)$ proceeds as outlined in Ref. 12]. The full cross section consists of three contributions. The first one contains all the channels with only gluons or light quarks in the initial state and $c$-quark fragmentation. Only this contribution carries explicit $m$ dependence. Second, this contribution must be extended by allowing for $c$ quarks in the initial state. The third contribution is due to gluon or lightquark fragmentation. The second and third contributions are calculated in the ZM-VFN scheme using the hard-scattering cross sections derived in Ref. 21]. A certain part of these contributions is due to Feynman diagrams with internal $c$-quark lines; another one is due to diagrams with external $c$-quark lines and contains $m$-dependent logarithms, which are resummed. Thus, in the FFN scheme, the $m$ dependence of these contributions would only enter beyond NLO, which is reflected in the ZM-VFN scheme by the generic suppression of the $c$-quark PDF relative to the gluon and light-quark ones and of the gluon and light-quark 
FFs relative to the $c$-quark one. This entitles us to omit this $m$ dependence by calculating the $c$-quark-initiated contributions and those involving the fragmentation of gluons or light quarks in the ZM-VFN scheme. It turns out that the light-quark fragmentation contributions are negligible. However, gluon fragmentation contributes approximately $40 \%$ to the cross section, almost independent of $p_{T}$. For the $D^{*+}$ case, we showed in Ref. 12] that the effect of the $m$-dependent terms is much reduced in the full cross section, since those parts that have to be calculated with $m=0$ dominate. In fact, this observation carries over to the other charmed mesons considered here.

We are now in a position to present our numerical results for the cross sections of inclusive $D^{0}, D^{+}, D^{*+}$, and $D_{s}^{+}$hadroproduction to be directly compared with the CDF data [1], which come as distributions $d \sigma / d p_{T}$ at $\sqrt{S}=1.96 \mathrm{TeV}$ with $y$ integrated over the range $|y| \leq 1$. For each $X_{c}$ species, the particle and antiparticle contributions are averaged. We work in the GM-VFN scheme with $n_{f}=4$, thus excluding $X_{c}$ hadrons from $X_{b}$-hadron decays, which are vetoed in the CDF analysis [1]. We set $m=1.5 \mathrm{GeV}$ and evaluate $\alpha_{s}^{\left(n_{f}\right)}\left(\mu_{R}\right)$, where $\mu_{R}$ is the renormalization scale, with $\Lambda \frac{(4)}{\mathrm{MS}}=328 \mathrm{MeV}$ [22], corresponding to $\alpha^{(5)}\left(m_{Z}\right)=0.1181$. We employ proton PDF set CTEQ6.1M from the CTEQ Collaboration 22] and the FFs introduced above. We distinguish between the initial- and final-state factorization scales, $\mu_{F}$ and $\mu_{F}^{\prime}$, so that we have three unphysical mass scales altogether. Our default choice is $\mu_{R}=\mu_{F}=\mu_{F}^{\prime}=m_{T}$, where $m_{T}=\sqrt{p_{T}^{2}+m^{2}}$ the transverse mass. In order to conservatively estimate the theoretical error due to the scale uncertainty, we independently vary the values of $\mu_{R} / m_{T}, \mu_{F} / m_{T}$, and $\mu_{F}^{\prime} / m_{T}$ between $1 / 2$ and 2 , and determine the maximum upward and downward deviations from our default predictions.

Our theoretical predictions are compared with the CDF data on an absolute scale in Fig. 1] and in the data-over-theory representation with respect to our default results in Fig. 2. The four frames in each figure refer to $D^{0}, D^{+}, D^{*+}$, and $D_{s}^{+}$mesons. In all cases, we find good agreement in the sense that the theoretical and experimental errors overlap, i.e. the notorious discrepancy between experiment and theory [1] mentioned in the introduction has disappeared. In fact, our theoretical predictions provide the best description of the CDF data obtained so far.

As for the $D^{0}, D^{*+}$, and $D_{s}^{+}$mesons, many of the central data points fall into the theoretical error band, while those for the $D^{+}$mesons lie somewhat above it. With the exception of the $D_{s}^{+}$case, the experimental results are gathered on the upper side of the 


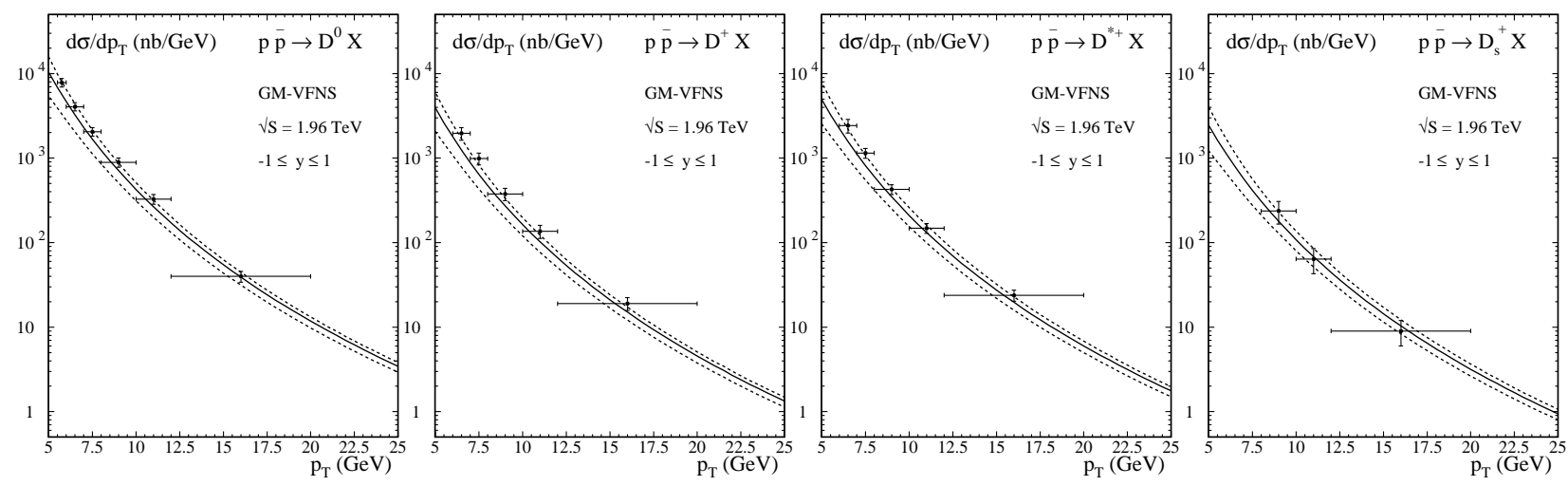

FIG. 1: Comparison of the CDF data 1] with our NLO predictions for $X_{c}=D^{0}, D^{+}, D^{*+}, D_{s}^{+}$. The solid lines represent our default predictions, while the dashed lines indicate the scale uncertainty (see text).
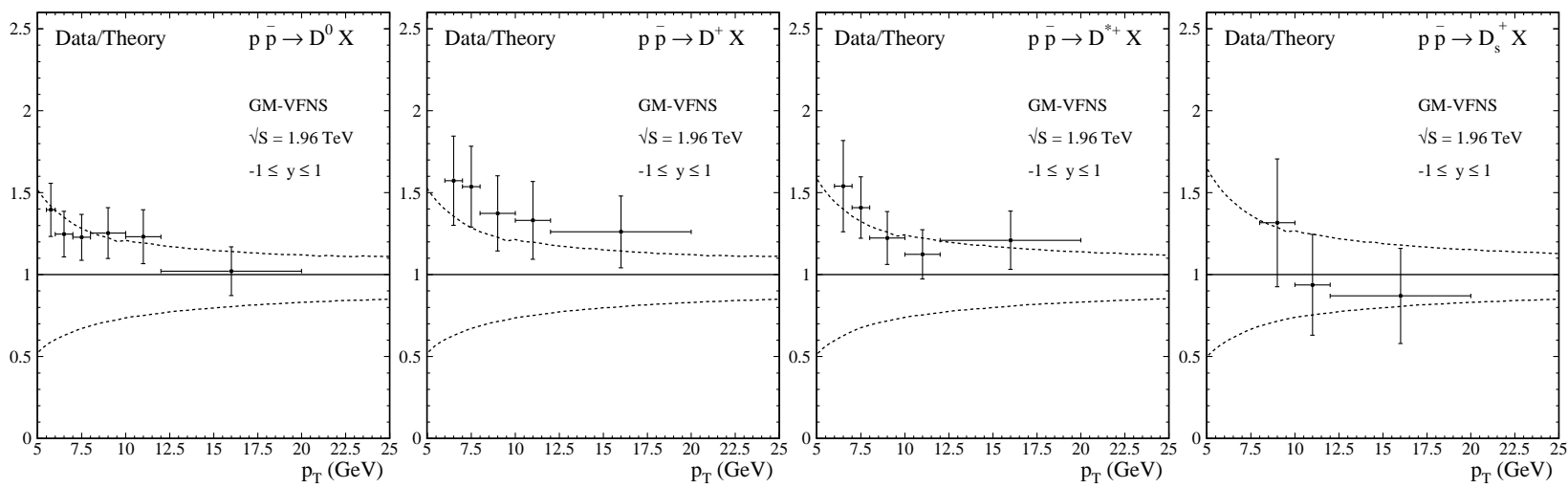

FIG. 2: Data-over-theory representation of Fig. 1 with respect to our default predictions.

theoretical error band, corresponding to a small value of $\mu_{R}$ and large values of $\mu_{F}$ and $\mu_{F}^{\prime}$, the $\mu_{R}$ dependence being dominant in the upper $p_{T}$ range. As is evident from Fig. 2, in these cases, the central data points tend to overshoot the central QCD predictions by a factor of about 1.5 at the lower end of the considered $p_{T}$ range, where the errors are largest, however. This factor is rapidly approaching unity as the value of $p_{T}$ is increased. The tendency of measurements of inclusive hadroproduction in Tevatron run II to prefer smaller renormalization scales is familiar from single jets, which actually favor $\mu_{R}=p_{T} / 2$ [23].

The overwhelming bulk of the theoretical error stems from the scale uncertainty discussed above. Residual sources of theoretical uncertainty include the variations in the value of $m$ and the adopted PDF and FF sets. We now quantitatively study the impact of these variations relative to the typical example of our default prediction for $D^{*+}$ mesons. The 
generous variation of $m$ by $\pm 20 \%$, from 1.2 to $1.8 \mathrm{GeV}$, induces a shift in cross section of only $\pm 2 \%$ at $p_{T}=5 \mathrm{GeV}$, which rapidly decreases towards larger values of $p_{T}$ because the $m$-dependent terms are themselves reduced in size. Switching to the NLO proton PDF set MRST2004 of Martin, Roberts, Stirling, and Thorne [24], with $\Lambda \frac{(4)}{\mathrm{MS}}=347 \mathrm{MeV}$ and a more physical parameterization of the gluon distribution to produce a better description of the Tevatron inclusive jet data, produces a reduction ranging from $-15 \%$ at $p_{T}=5 \mathrm{GeV}$ to $-1 \%$ at $p_{T}=20 \mathrm{GeV}$. The theoretical uncertainty due to the FFs was estimated in Ref. [12] to be of order $10 \%$ or less in the $p_{T}$ range considered here, by comparing FF sets [4] fitted separately to slightly incompatible ALEPH [16] and OPAL [17] data.

In conclusion, the GM-VFN scheme, which we elaborated at NLO for the inclusive photo11] and hadroproduction [12, 13] of heavy-flavored hadrons, resums large logarithms by the DGLAP evolution of non-perturbative FFs and guarantees the universality of the latter as in the ZM-VFN scheme and simultaneously retains the $m$-dependent terms of the FFN scheme without additional theoretical assumptions. Adopting this framework in combination with new fits of $D^{0}, D^{+}, D^{*+}$, and $D_{s}^{+}$FFs to OPAL data from LEP1 [17, 19], we managed for the first time to reconcile the CDF data on the production of these mesons in Tevatron run II 1] with QCD within errors and thus eliminated a worrisome discrepancy. Furthermore, we presented the first NLO predictions for the $D_{s}^{+}$data [1].

\section{Acknowledgments}

The work of I.S. was supported by DESY. This work was supported in part by BMBF Grant No. 05 HT4GUA/4.

[1] CDF Collaboration, D. Acosta et al., Phys. Rev. Lett. 91, 241804 (2003).

[2] Calculation as in Ref. [3] with $D^{*+}$ FFs of Ref. [4].

[3] B.A. Kniehl, G. Kramer, and B. Pötter, Nucl. Phys. B597, 337 (2001).

[4] J. Binnewies, B.A. Kniehl, and G. Kramer, Phys. Rev. D 58, 014014 (1998).

[5] M. Cacciari and P. Nason, JHEP 0309, 006 (2003).

[6] CDF Collaboration, F. Abe et al., Phys. Rev. D 50, 4252 (1994); Phys. Rev. Lett. 75, 1451 (1995); CDF Collaboration, D. Acosta et al., Phys. Rev. D 65, 052005 (2002). 
[7] E.L. Berger, B.W. Harris, D.E. Kaplan, Z. Sullivan, T.M.P. Tait, and C.E.M. Wagner, Phys. Rev. Lett. 86, 4231 (2001).

[8] M. Cacciari and M. Greco, Nucl. Phys. B421, 530 (1994).

[9] W. Beenakker, H. Kuijf, W.L. van Neerven, and J. Smith, Phys. Rev. D 40, 54 (1989); P. Nason, S. Dawson, and R.K. Ellis, Nucl. Phys. B327, 49 (1989); B335, 260(E) (1990); I. Bojak and M. Stratmann, Phys. Rev. D 67, 034010 (2003).

[10] M. Cacciari, M. Greco, and P. Nason, JHEP 9805, 007 (1998).

[11] G. Kramer and H. Spiesberger, Eur. Phys. J. C 22, 289 (2001); 28, 495 (2003); 38, 309 (2004).

[12] B.A. Kniehl, G. Kramer, I. Schienbein, and H. Spiesberger, Phys. Rev. D 71, 014018 (2005).

[13] B.A. Kniehl, G. Kramer, I. Schienbein, and H. Spiesberger, Eur. Phys. J. C 41, 199 (2005).

[14] M.A.G. Aivazis, J.C. Collins, F.I. Olness, and W.-K. Tung, Phys. Rev. D 50, 3102 (1994).

[15] F.I. Olness, R.J. Scalise, and W.-K. Tung, Phys. Rev. D 59, 014506 (1999).

[16] ALEPH Collaboration, R. Barate et al., Eur. Phys. J. C 16, 597 (2000).

[17] OPAL Collaboration, K. Ackerstaff et al., Eur. Phys. J. C 1, 439 (1998).

[18] B.A. Kniehl and G. Kramer, Phys. Rev. D 71, 094013 (2005).

[19] OPAL Collaboration, G. Alexander et al., Z. Phys. C 72, 1 (1996).

[20] CLEO Collaboration, R.A. Briere et al., Phys. Rev. D 62, 072003 (2000); CLEO Collaboration, M. Artuso et al., ibid. 70, 112001 (2004).

[21] F. Aversa, P. Chiappetta, M. Greco, and J.Ph. Guillet, Phys. Lett. B 210, 225 (1988); 211, 465 (1988); Nucl. Phys. B327, 105 (1989).

[22] CTEQ Collaboration, J. Pumplin et al., JHEP 0207, 012 (2002); CTEQ Collaboration, D. Stump et al., ibid. 0310, 046 (2003).

[23] R. Field, for the CDF Collaboration, in Proceedings of the XIII ${ }^{\text {th }}$ International Workshop on Deep Inelastic Scattering (DIS05), Madison, Wisconsin, 2005 (American Institute of Physics, Melville, to be published); B. Davies, for the D0 Collaboration, ibid.

[24] A.D. Martin, R.G. Roberts, W.J. Stirling, and R.S. Thorne, Phys. Lett. B 604, 61 (2004). 